Int. J. Odontostomat., 6(1):89-95, 2012.

\title{
Instrumentación Rotatoria en Endodoncia: Reporte de Casos Clínicos
}

\author{
Rotary Instrumentation in Endodontics: Clinical Cases Report
}

José Leonardo Jiménez Ortiz* \& Teresita Marisol Del Río Cazares**

JIMÉNEZ, O. J. L. \& DEL RIO, C. T. M. Instrumentación rotatoria en endodoncia: reporte de casos clínicos. Int. J. Odontostomat., 6(1):89-95, 2012.

RESUMEN: Los recientes avances en endodoncia de los últimos años, como la introducción de instrumentación rotatoria con limas de Niquel Titanio (NiTi), han simplificado el proceso del tratamiento, mejorando el éxito a largo plazo en términos de tiempo, efectividad y reducción de riesgos, comparados con la instrumentación manual con limas de acero inoxidable. Sin embargo, para algunos clínicos resulta difícil seleccionar el sistema rotatorio NiTi más apropiado, debido a la numerosa cantidad de instrumentos que conforman los diferentes sistemas existentes en el mercado. El presente reporte, basado en casos clínicos de la práctica diaria, tiene como objetivo mostrar, analizar y destacar la posibilidad de realizar tratamientos endodónticos predecibles con la técnica corono apical utilizando el sistema ProTaper Universal.

PALABRAS CLAVE: endodoncia, instrumentación rotatoria, técnica corono apical, ProTaper Universal.

\section{INTRODUCCIÓN}

El principal objetivo del tratamiento de canales es la limpieza mecánica y química de la cavidad pulpar y su obturación tridimensional con un material de sellado inerte y un sellado coronal que prevenga el ingreso de microorganismos (Schilder, 1974).

Los avances científicos de los últimos años, han permitido lograr este objetivo de manera más eficiente, mejorado así el éxito a largo plazo del tratamiento endodóntico. El uso de limas rotatorias de níquel titanio (NiTi) durante la instrumentación, ha ganado popularidad tanto en los endodoncistas como en los dentistas de practica general, debido a su mayor flexibilidad (Walia et al., 1988; Parashos \& Messer 2006) y capacidad de mantener la configuración original de los canales curvos con paredes delgadas (Aguiar et al., 2009). Además, existe evidencia de que estos sistemas reducen las fallas relacionadas con la instrumentación y permiten una conformación de conicidad adecuada con gran velocidad y efectividad (Guelzow et al., 2005; Moore et al., 2009). A pesar de presentar estas ventajas en comparación con la instrumentación manual realizada con limas de acero inoxidable, para algunos clínicos resulta difícil seleccionar el sistema rotatorio de limas NiTi más apropiado, debido a la numerosa cantidad de instrumentos que conforman los diferentes sistemas existentes en el mercado.

El sistema Protaper Universal (Dentsply Maillefer, Baillegues, Suiza) presenta en el diseño de sus instrumentos, una sección transversal triangular convexa, con una punta no cortante. Consta de seis limas, tres de conformación: SX, S1 y S2; y tres limas de acabado: F1, F2, F3, además de otras dos limas accesorias de acabado: F4 y F5. De acuerdo con las instrucciones del fabricante, la lima SX se utiliza para conformar la porción coronal, la lima S1 se emplea inicialmente $4 \mathrm{~mm}$ menos de la LT establecida, mientras que la $S 1$ y S2 se llevan a LT para ensanchar progresivamente el tercio apical. Las limas F1, F2 y $\mathrm{F} 3$, son utilizadas para completar la terminación apical. Las limas F4 y F5 se usan solamente si el caso lo requiere (West, 2006).

El objetivo de este reporte es presentar y analizar casos clínicos de la práctica diaria, para eviden-

\footnotetext{
- Cirujano Dentista Especialista en Endodoncia, Jefe de Investigación, Escuela de Odontología, Facultad de Ciencias de la Salud, Universidad de Montemorelos, Montemorelos Nuevo León, México.

* Cirujano Dentista Especialista en Endodoncia, Práctica privada en Piedras Negras, Coahuila, México.
} 
ciar la eficacia en el tratamiento endodóntico de órganos dentales posteriores mandibulares y maxilares, utilizando la técnica corono apical con los instrumentos rotatorios NiTi ProTaper Universal.

\section{REPORTE DE CASOS}

Se presentan cuatro casos clínicos atendidos endodónticamente por los autores, los cuales fueron instrumentados y obturados en una sola sesión, utilizando el sistema ProTaper Universal. En cada uno de los casos, previa anestesia local, se procedió al aislamiento absoluto con dique de hule para llevar a cabo el acceso coronal adecuado. Con la ayuda de lupas Surgitel (General Scientific Corporation, USA) con magnificación 3.0 X, se localizaron cada uno de los orificios de entrada de los canales radiculares, y se procedió a establecer la longitud de trabajo (LT) en cada uno de los canales de los diferentes órganos dentales, utilizando el localizador de foramen apical Root ZX (J. Morita Inc, USA). Todos los canales se conformaron con la técnica corono apical con el sistema de instrumentación rotatoria ProTaper Universal (Dentsply Maillefer, Baillegues, Suiza) de acuerdo a las instrucciones del fabricante: limas SX, S1 y S2 para la conformación del tercio coronal y medio, y limas F1, F2 y F3 para la preparación del tercio apical. Se permeabilizó en los todos canales previamente instrumentados, con lima tipo $\mathrm{K}$ No. 10 (Dentsply Maillefer, Baillegues, Suiza).

Durante la preparación biomecánica, se irrigó abundantemente con hipoclorito de sodio al $2.5 \%$, EDTA al $17 \%$ (Vista Dental Products, USA) y solución salina al final, realizando activación sónica con el sistema Endoactivator (Tulsa Dental Specialties, USA) de acuerdo a las instrucciones del fabricante, al introducir con movimientos verticales en cada canal, una punta amarilla 20/.04, roja 25/.04, o azul 30/.04 dependiendo del calibre antes instrumentado. Esto se llevó a cabo $3 \mathrm{~mm}$ cortos de la LT establecida, en velocidad media durante 30 segundos. Se procedió al secado de los canales con puntas de papel estéril. La obturación se realizó con la técnica de cono único, empleando gutapercha ProTaper Universal F1, F2 o F3 (Dentsply Maillefer, Baillegues, Suiza) según el calibre de la última lima utilizada durante la instrumentación de los canales en cada uno de los casos, utilizando cemento sellador Sealapex (SybronEndo, USA). La cavidad de acceso se selló con Provisit (Casa Idea, México) y los pacientes fueron remitidos a sus respectivos dentistas tratantes para la restauración protésica definitiva.

\section{CASO 1}

Paciente femenino de 28 años de edad, sintomático que acude a consulta endodóntica de urgencia por el primer molar inferior izquierdo (3.6). Sin datos importantes en su historia médica, al interrogatorio refirió sentir dolor con evolución de una semana. A la exploración se observó presencia de tejido dental reblandecido en la superficie oclusal. No existía evidencia de inflamación de tejidos blandos o movilidad dental. Radiográficamente, se pudo apreciar una zona radiolúcida a nivel coronal, con proximidad al cuerno distal de la cámara pulpar, con ligero ensanchamiento de ligamento periodontal, así como dos raíces sin obstrucción aparente en sus canales radiculares (Fig. 1). Basados en los hallazgos subjetivos y objetivos, se estableció el diagnóstico de

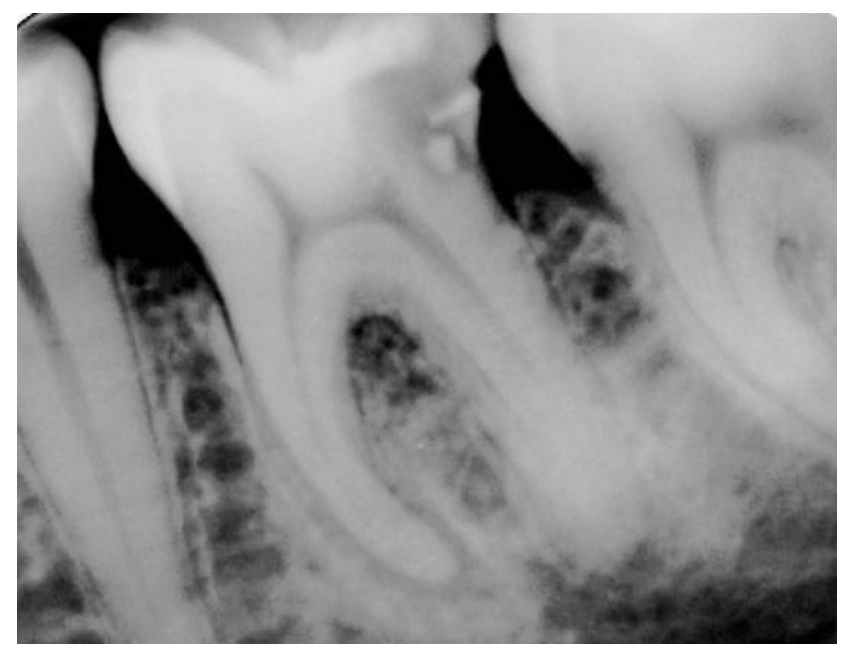

Fig. 1. Radiografía inicial del órgano dental 3.6

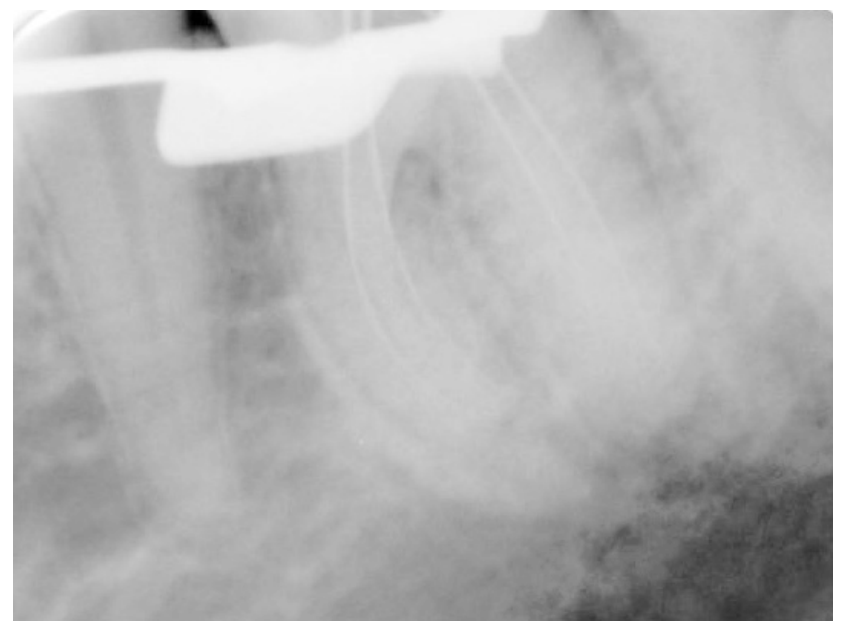

Fig. 2. Radiografía mesioradial evidenciando la longitud de trabajo establecida en los cuatro conductos: mesiovestibular, mesiolingual, distovestibular y distolingual. 


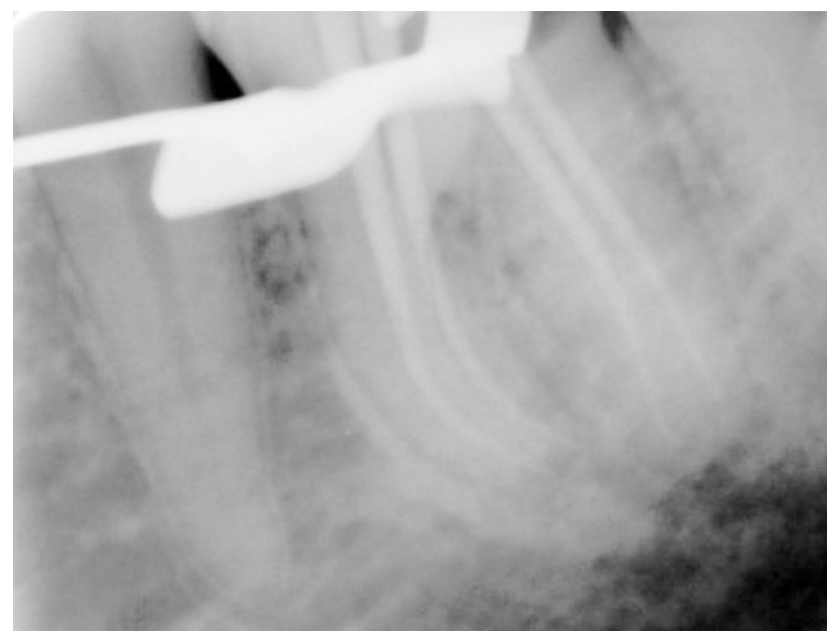

Fig. 3. Radiografía mesioradial donde se aprecia la prueba de punta en los conductos de la raíz mesial y distal.

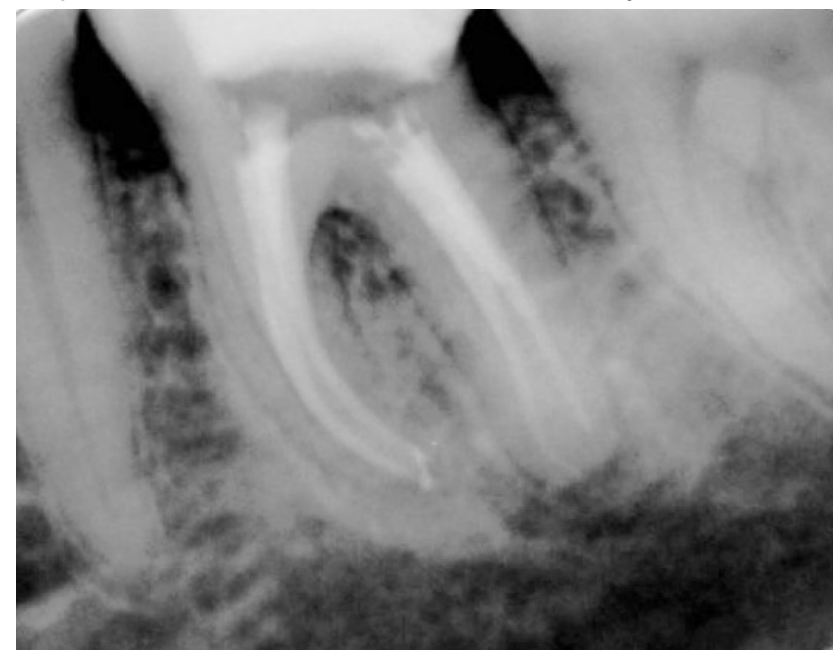

Fig. 4. Radiografía final de obturación con la técnica de cono único. Extrusión de cemento sellador en la región apical en la raíz mesial que verifica la patencia apical de los conductos.

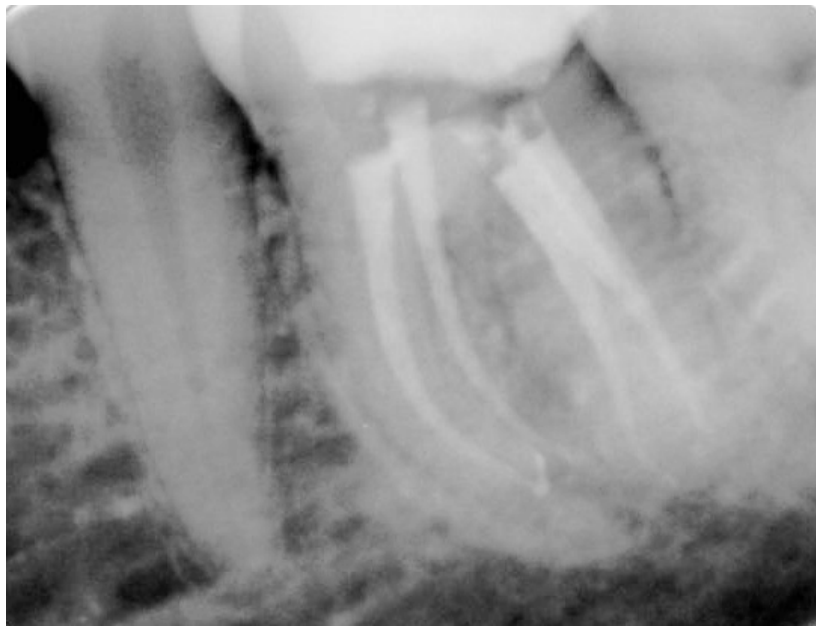

Fig. 5. Radiografía mesioradial mostrando la conformación y obturación cónica, continua y uniforme lograda con el Sistema ProTaper Universal en todo el sistema de conductos radiculares.
Pulpitis Irreversible del diente 3.6, y se procedió a realizar la terapia endodóntica con el Sistema ProTaper Universal de la manera descrita anteriormente, localizando cuatro canales, dos en la raíz mesial: el mesiovestibualr y mesiolingual; dos canales más en la raíz distal: el distovestibualr y distolingual (Fig. 2), instrumentando hasta el calibre F2 en los cuatro canales (Fig. 3), realizando la obturación con gutapercha del mismo calibre (Figs. 4 y 5 ).

\section{CASO 2}

Paciente masculino de 52 años de edad, referido para recibir tratamiento endodóntico, en el tercer molar inferior izquierdo (3.8). Sin datos importantes en su historia médica, asintomático, no presentó respuesta a los estímulos térmicos. Al interrogatorio refirió haber sido enviado por su dentista por motivos protésicos. A la exploración se observó la presencia de obturación defectuosa con amalgama en la superficie oclusal. No existía evidencia de inflamación de tejidos blandos o movilidad dental. Radiográficamente se pudo apreciar, inclinación mesial del órgano dental a tratar, una zona radiopaca a nivel coronal, sin proximidad a la cámara pulpar, una sola raíz sin obstrucción aparente en su canal radicular, presentado una curvatura moderada en el tercio apical (Fig. 6). Se estableció el diagnóstico de Pulpa Sana del diente 3.8, y se procedió realizar el tratamiento endodóntico por motivos protésicos con el Sistema ProTaper Universal, instrumentando hasta el calibre F3 el único canal localizado, para realizar su posterior obturación con gutapercha del mismo calibre (Fig. 7).

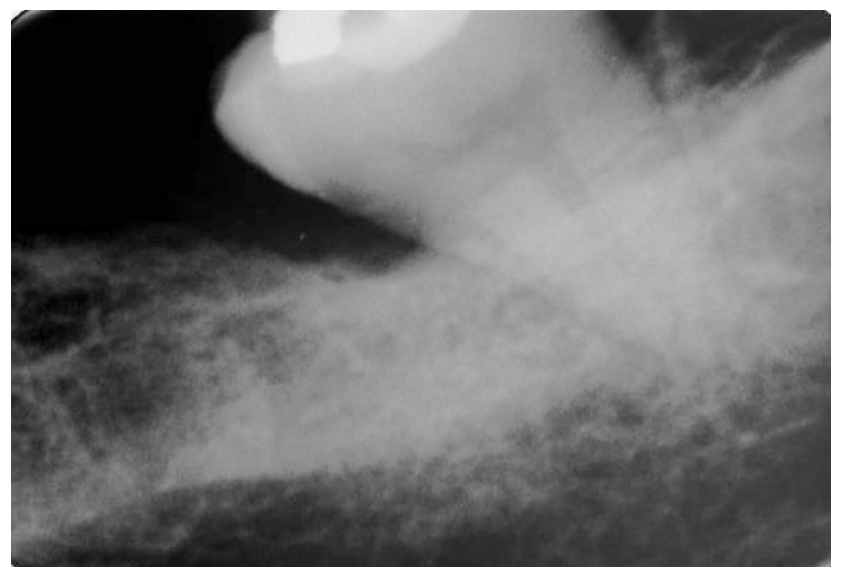

Fig. 6. Radiografía inicial del órgano dental 3.8 donde se aprecia la curvatura de la región apical. 


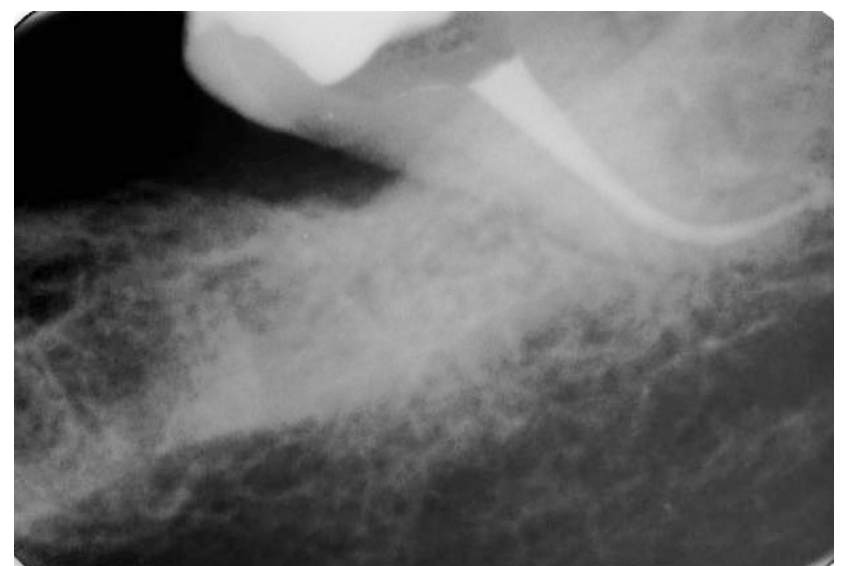

Fig. 7.Radiografía final de obturación conservando y respetando la configuración anatómica original del conducto, con presencia de extrusión de cemento sellador en la región apical, verificando la patencia apical del conducto.

\section{CASO 3}

Paciente masculino de 33 años de edad, se presenta asintomático para recibir valoración endodóntica en el primer molar superior izquierdo (1.6). Sin datos importantes en su historia médica, presentó respuesta a los estímulos térmicos. Al interrogatorio no refirió sentir dolor, solamente sensibilidad al ingerir bebidas frías. A la exploración se observó una cavidad pigmentada en la superficie oclusal. No existía evidencia de inflamación de tejidos blandos o movilidad dental. Radiográficamente, se pudo apreciar una zona radiolúcida a nivel coronal, con tres raíces separadas sin obstrucción aparente en sus canales radiculares (Fig. 8). Basados en los hallazgos subjetivos y objetivos, se estableció el diagnóstico de Pulpitis Irreversible del diente 1.6, y se procedió a realizar la terapia endodóntica con el Sistema

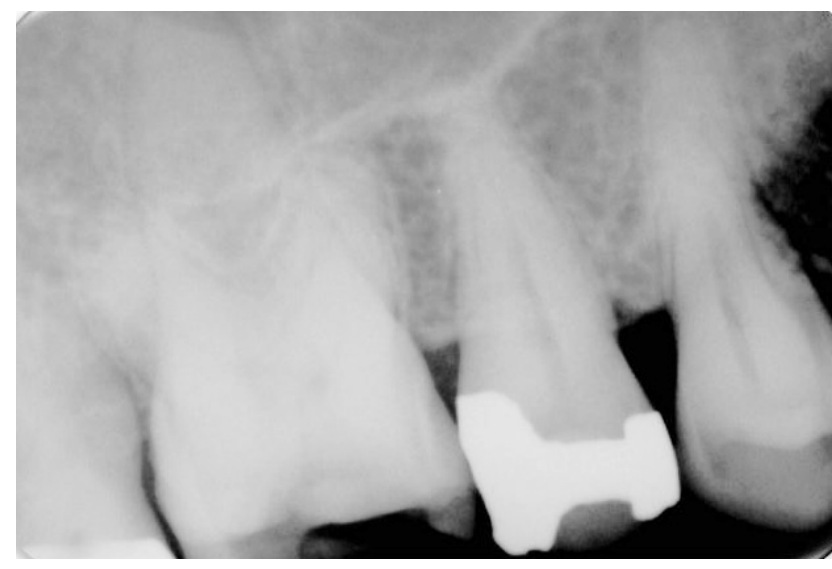

Fig. 8. Radiografía inicial órgano dental 1.6.
ProTaper Universal de la manera descrita anteriormente, localizando cuatro canales, dos en la raíz mesiovestibular, uno en la raíz distovestibular y otro más en la raíz palatina. Se instrumentó el canal mesiovestibular hasta el claibre $\mathrm{F} 2$, el cuarto canal hasta F1, el canal distovestibular hasta F2, mientras que el canal palatino hasta, F2 (Fig. 9), para llevar a cabo la obturación con gutapercha del mismo calibre (Fig. 10).

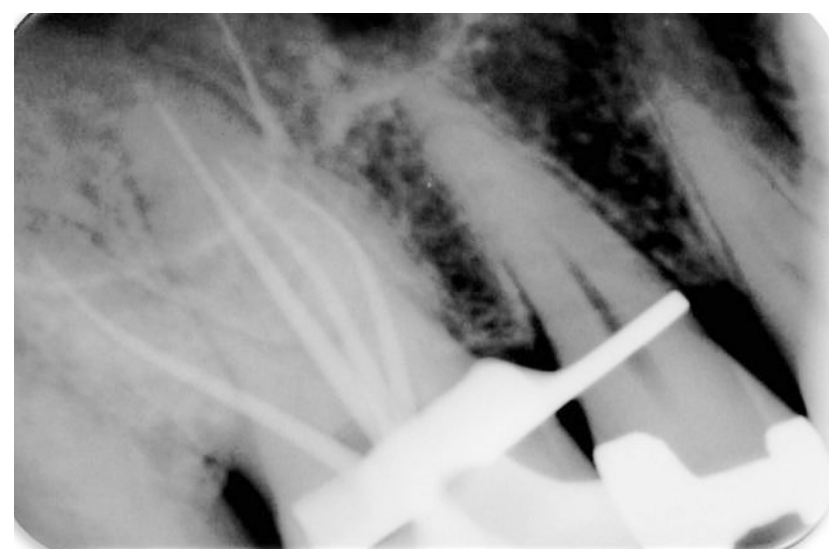

Fig. 9. Radiografía mesioradial con la prueba de punta, donde se evidencia la conformación de los conductos en las tres raíces.

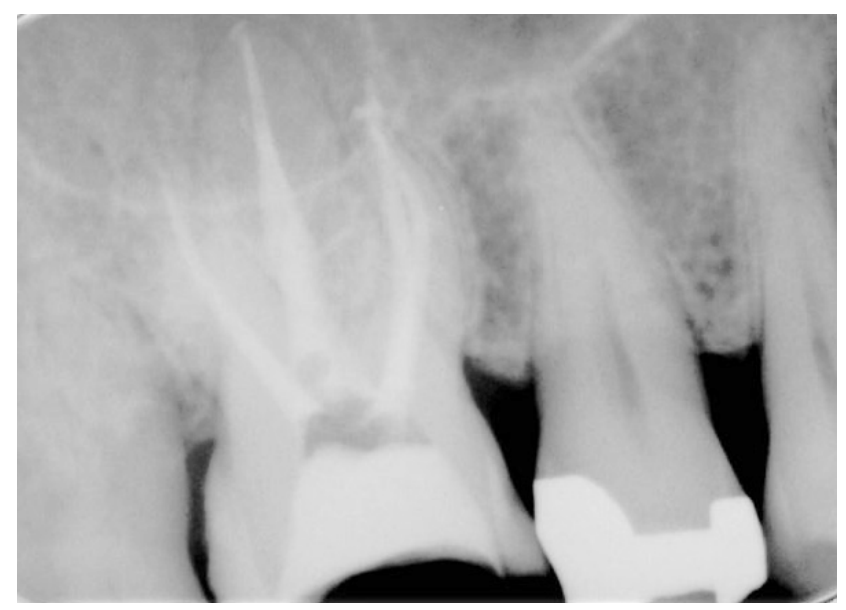

Fig. 10. Radiografía final con la obturación de los cuatro conductos: mesiovestibular, cuarto conducto, distovestibular y palatino. Extrusión de cemento sellador en la región apical en la raíz mesial y palatina que verifica la patencia apical de los conductos.

\section{CASO 4}

Paciente femenino de 74 años de edad, referida por su dentista tratante a consulta endodóntica del primer premolar superior derecho (2.4). Sin datos im- 
portantes en su historia médica, presentó respuesta a los estímulos térmicos. Al interrogatorio refirió sentir sensibilidad marcada al ingerir bebidas frías con evolución de dos semanas, esto inmediatamente después de ser tratada protésicamente en ese diente. A la exploración se observó la presencia de una corona de metal porcelana. No existía evidencia de inflamación de tejidos blandos o movilidad dental. Radiográficamente se pudo apreciar una zona radiopaca a nivel coronal con una área radiolúcida de la zona mesial en interproximal, que evidenciaba la falta de sellado de la prótesis. Se observó también, la presencia dos raíces separadas: una vestibular y otra palatina, con obstrucción evidente debido a la calcificación de sus canales radiculares, presentado una curvatura moderada en el tercio apical de su raíz vestibular (Fig. 11). Una vez establecido el diagnóstico de Pulpitis Irreversible del diente 2.4 y previa anestesia local, la corona fue removida para proceder al aislamiento absoluto y localización inicial de los ca-

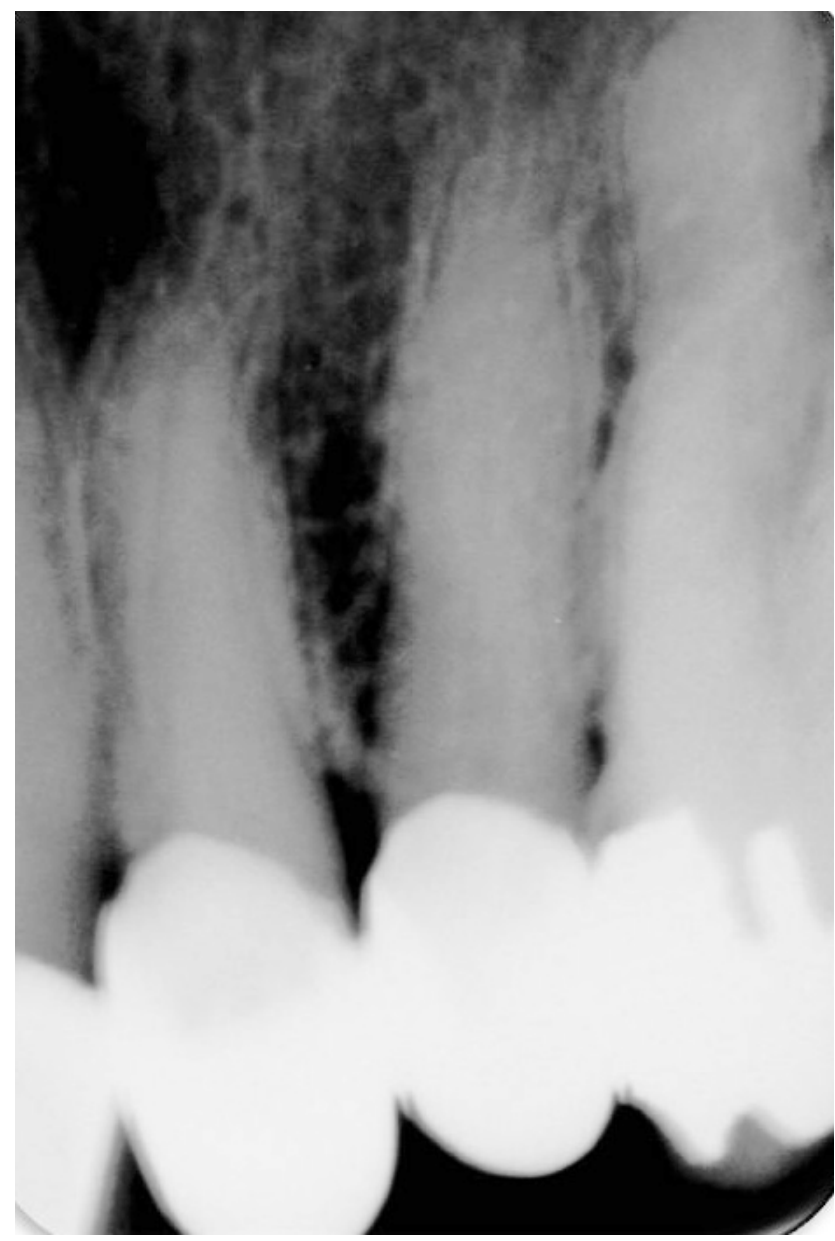

Fig. 11. Radiografía inicial órgano dental 2.4, que evidencia la calcificación de los conductos en su raíz vestibular y palatina. nales vestibular y palatino con limas de acero inoxidable No. 6. Después de haber logrado explorar la trayectoria de los canales con esa lima, se procedió a instrumentar con limas de calibre pequeño: No.8, No. 10 y No. 15, hasta lograr un camino guía reproducible, para dar paso a la instrumentación con el Sistema ProTaper Universal de la manera descrita anteriormente hasta el calibre F2 en ambos canales. La obturación se llevó a cabo utilizando gutapercha del mismo sistema y calibre (Fig. 12).

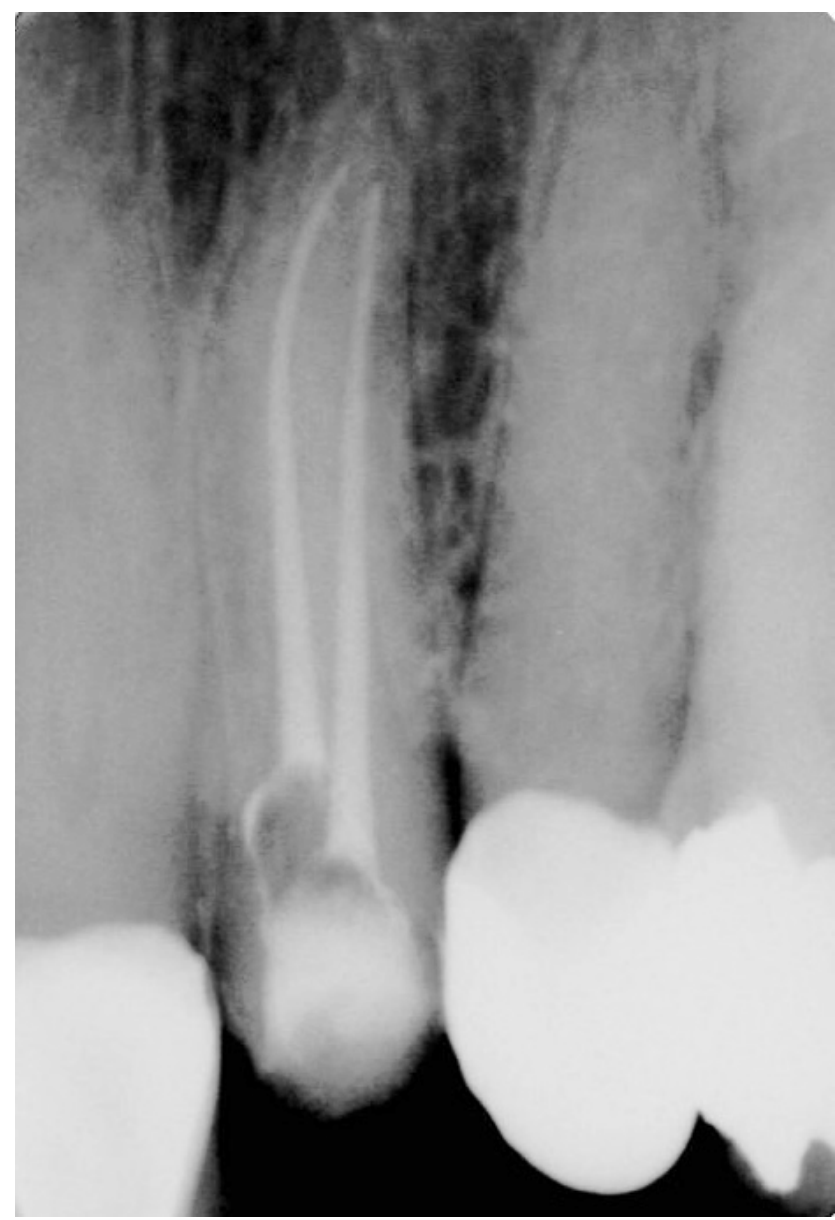

Fig. 12. Radiografía final de obturación donde se logró la conformación y obturación de los conductos vestibular y palatino, que presentaban calcificación en su trayecto.

\section{DISCUSIÓN}

La instrumentación rotatoria con limas de níquel titanio (NiTi), es un importante coadyuvante en la terapia endodóntica, ya que ha mejorado la práctica clínica en términos de tiempo, efectividad y reducción de riesgos (Parashos \& Messer) comparados con la instrumentación manual con limas de ace- 
ro inoxidable. Resulta difícil para algunos clínicos seleccionar el sistema rotatorio NiTi más adecuado a sus necesidades, debido a la numerosa cantidad de instrumentos que conforman los diferentes sistemas que actualmente se encuentran a la venta en el mercado endodóntico, aunado a la dificultad de familiarizarse con la secuencia de uso de los instrumentos y a la gran inquietud existente respecto a la fractura de estos instrumentos. Se ha publicado en la literatura de los últimos diez años, reportes de estudios que muestran hallazgos referentes a los diversos factores que influyen en la fractura (Martín et al., 2003; Shen et al., 2009), resistencia a la fatiga cíclica (Bahia et al., 2008; Fife et al., 2004), facilidad de uso (Peru et al., 2006) y capacidad de configuración los canales radiculares (Hülsmann et al., 2003; Williamson et al., 2009) de los diferentes sistemas de instrumentación rotatoria NiTi. Existe evidencia que el sistema Protaper Universal (Dentsply Maillefer, Baillegues, Suiza), el cual presenta en el diseño de sus instrumentos NiTi una conicidad variable progresiva, con una sección transversal triangular convexa y punta no cortante, mejora la acción de corte, flexibilidad y eficiencia (Ozgur Uyanik et al., 2006), disminuye la fricción rotacional entre la hoja de corte de la lima y dentina, minimizando la fatiga del instrumento (Calberson et al., 2004). La separación de limas se presenta como un riesgo potencial cuando se utiliza cualquier sistema de instrumentación NiTi durante la conformación de canales radiculares en la terapia endodóntica. La resistencia a la fatiga cíclica de la limas NiTi del sistema ProTaper Universal (Dentsply Maillefer, Baillegues, Suiza), ha sido examinada en estudios in vivo, después de utilizarse de manera inicial en canales rectos y curvos demostrando resultados favorables (Ounsi et al., 2007). Se ha demostrado que el uso clínico prolongado de los instrumentos rotatorios ProTaper (de 12 hasta 16 canales) redujo significativamente su resistencia a la fatiga cíclica (Fife et al.). Reportes indican que las limas ProTaper pueden ser utilizadas de forma segura por lo menos hasta cuatro veces (Wolcott et al., 2006). Estudios realizados sugieren que el sistema ProTaper presenta una mayor capacidad de crear una preparación más regular (Guelzow et al.), produciendo configuraciones centradas en canales radiculares curvos y delgados con una baja proporción de desviación apical (Aguiar et al.), al ser comparados con otros sistemas de instrumentación. Existe evidencia de que una vez terminada la preparación mecánica, se debe complementar la limpieza de los canales radiculares mediante el uso de las diferentes técnicas de activación de la solución irrigante (Gu et al., 2009). El sistema EndoActivator (Dentsply Tulsa Dental Specialties, USA) de reciente introducción como coadyuvante durante la terapia endodóntica, consiste en una pieza de mano inalámbrica que utiliza tres puntas desechables de polímero flexible con diferentes calibres no cortantes, diseñadas para la activación sónica de los agentes irrigantes dentro del sistema de canales radiculares, produciendo una agitación vigorosa de fluídos (Ruddle, 2008). Este sistema ha demostrado proporcionar una mejor irrigación en canales laterales simulados a 4,5 y $2 \mathrm{~mm}$ de la longitud de trabajo, comparado con el solo uso tradicional de irrigación con jeringa (de Gregorio et al., 2009), así como la remoción del lodo dentinario con agentes desmineralizantes como el EDTA y películas de biofilm en canales radiculares curvos (Caron et al., 2010).

En conclusión, el presente reporte de órganos dentales posteriores mandibulares y maxilares evidencia la eficacia clínica de una práctica endodóntica predecible, utilizando la técnica corono apical con los instrumentos rotatorios NiTi ProTaper Universal.

JIMÉNEZ, O. J. L. \& DEL RIO, C. T. M. Rotary instrumentation in endodontics: clinical cases report. Int. J. Odontostomat., 6(1):89-95, 2012.

ABSTRACT: Advances in recent years in endodontics, such introduction of rotary Niquel Titanium (NiTi) instrumentation have simplified the treatment process and improved the long term success in terms of procedural time, accuracy, and risk reduction compared with the previously used, manual, stainless steel files. However, selecting the most appropriate NiTi system remains difficult for clinicians because of the large number of NiTi rotary instruments available nowadays on the market. This paper aims to show, analyze and emphasize, on the basis of every day clinical practice, the possibility of realizing predictability root canal preparations with the crown down technique of ProTaper Universal system.

KEY WORDS: root canal treatment, rotary instrumentation, crown-down technique, Protaper Universal. 


\section{REFERENCIAS BIBLIOGRÁFICAS}

Aguiar, C. M.; de Andrade Mendes, D.; Câmara, A. C. \& de Figueiredo, J. A. Evaluation of the centering ability of the ProTaper Universal rotary system in curved roots in comparison to Nitiflex files. Aust. Endod. J., 35(3):174-9, 2009.

Bahia, M. G.; Melo, M. C. \& Buono, V. T. Influence of cyclic torsional loading on the fatigue resistance of $\mathrm{K} 3$ instruments. Int. Endod. J., 41(10):883-91, 2008.

Calberson, F. L.; Deroose, C. A.; Hommez, G. M. \& De Moor, R. J. Shaping ability of ProTaper nickel titanium files in simulated resin root canals. Int. Endod. J., 37(9):613-23, 2004.

Caron, G.; Nham, K.; Bronnec, F. \& Machtou, P. Effectiveness of different final irrigant activation protocols on smear layer removal in curved canals. J. Endod., 36(8):1361-6, 2010.

de Gregorio, C.; Estevez, R.; Cisneros, R.; Heilborn, C. \& Cohenca, N. Effect of EDTA, sonic, and ultrasonic activation on the penetration of sodium hypochlorite into simulated lateral canals: an in vitro study. J. Endod., 35(6):891-5, 2009.

Fife, D.; Gambarini, G. \& Britto, L. R. Cyclic fatigue testing of ProTaper NiTi rotary instruments after clinical use. Oral Surg. Oral Med. Oral Pathol. Oral Radiol. Endod., 97(2):251-6, 2004

Gu, L. S.; Kim, J. R.; Ling, J.; Choi, K. K.; Pashley, D. H. \& Tay, F. R. Review of contemporary irrigant agitation techniques and devices. J. Endod., 35(6):791-804, 2009.

Guelzow, A.; Stamm, O.; Martus, P. \& Kielbassa, A. M. Comparative study of six rotary nickel-titanium systems and hand instrumentation for root canal preparation. Int. Endod. J., 38(10):743-52, 2005.

Hülsmann, M.; Herbst, U. \& Schäfers, F. Comparative study of root-canal preparation using Lightspeed and Quantec SC rotary NiTi instruments. Int. Endod. J., 36(11):748-56, 2003.

Martín, B.; Zelada, G.; Varela, P.; Bahillo, J. G.; Magán, F.; Ahn, S. \& Rodríguez, C. Factors influencing the fracture of nickeltitanium rotary instruments. Int. Endod. J., 36(4):262-6, 2003.

Moore, J.; Fitz-Walter, P. \& Parashos, P. A micro-computed tomographic evaluation of apical root canal preparation using three instrumentation techniques. Int. Endod. J., 42(12):1057-64, 2009.

Ounsi, H. F.; Salameh, Z.; Al-Shalan, T.; Ferrari, M.; Grandini, S.; Pashley, D. H. \& Tay, F. R. Effect of clinical use on the cyclic fatigue resistance of ProTaper nickel-titanium rotary instruments. J. Endod., 33(6):737-41, 2007.

Ozgur Uyanik, M.; Cehreli, Z. C.; Ozgen Mocan, B. \& Tasman Dagli, F. Comparative evaluation of three Nickel-titanium instrumentation systems in human teeth using computed tomography. J. Endod., 32(7):668-71, 2006.
Parashos, P. \& Messer, H. H. The diffusion of innovation in dentistry: a review using rotary nickel-titanium technology as an example. Oral Surg. Oral Med. Oral Pathol. Oral Radiol. Endod., 101(3):395-401, 2006.

Peru, M.; Peru, C.; Mannocci, F.; Sherriff, M.; Buchanan, L. S. \& Pitt Ford, T. R. Hand and nickel-titanium root canal instrumentation performed by dental students: a microcomputed tomographic study. Eur. J. Dent. Educ., 10(1):529, 2006.

Ruddle, C. J. Endodontic disinfection: tsunami irrigation. Endod. Practice, 11:7-15, 2008.

Schilder, H. Cleaning and shaping the root canal. Dent. Clin. North Am., 18(2):269-96, 1974.

Shen, Y.; Coil, J. M.; McLean, A. G.; Hemerling, D. L. \& Haapasalo, M. Defects in nickel-titanium instruments after clinical use, Part 5: single use from endodontic specialty practices. J. Endod., 35(10):1363-7, 2009.

Walia, H. M.; Brantley, W. A. \& Gerstein, H. An initial investigation of the bending and torsional properties of nitinol root canal files. J. Endod., 14(7):346-51, 1988.

West, J. Progressive taper technology: rationale and clinical technique for the new ProTaper universal system. Dent. Today, 25(12):64, 66-9, 2006.

Williamson, A. E.; Sandor, A. J. \& Justman, B. C. A comparison of three nickel titanium rotary systems, EndoSequence, ProTaper universal, and profile GT, for canal-cleaning ability. J. Endod., 35(1):107-9, 2009.

Wolcott, S.; Wolcott, J.; Ishley, D.; Kennedy, W.; Johnson, S.; Minnich, S. \& Meyers, J. Separation incidence of protaper rotary instruments: a large cohort clinical evaluation. J. Endod., 32(12):1139-41, 2006.

Dirección para correspondencia:

José Leonardo Jiménez Ortiz

Cirujano Dentista Especialista en Endodoncia, Jefe de Investigación, Escuela de Odontologia

Facultad de Ciencias de la Salud

Universidad de Montemorelos

Libertad No. 1300 Col. Centro

Montemorelos Nuevo León

C.P. 67530

MÉXICO

Teléfonos: +52 (826) 263-5824; +52(826) 263-5529

Email: endodoncia@um.edu.mx

Recibido : 22-11-2011 